\title{
Levosimendan in rats decreases acute kidney injury after cardiopulmonary resuscitation by improving mitochondrial dysfunction
}

\author{
Li Zhao, Lei Tian, Shiwei Wang, Weiqiang Yang, Xiaoye Lu, Changqing Zhu \\ Department of Emergency, Renji Hospital, School of Medicine, Shanghai Jiao Tong University, Shanghai, China \\ Contributions: (I) Conception and design: L Zhao, L Tian, C Zhu; (II) Administrative support: L Tian, C Zhu; (III) Provision of study materials or \\ patients: L Zhao, S Wang, W Yang; (IV) Collection and assembly of data: X Lu, C Zhu; (V) Data analysis and interpretation: L Zhao, X Lu, C Zhu; (VI) \\ Manuscript writing: All authors; (VII) Final approval of manuscript: All authors. \\ Correspondence to: Changqing Zhu; Tian Lei. Department of Emergency, Renji Hospital, School of Medicine, Shanghai Jiao Tong University, \\ Shanghai 200127, China. Email: zhucq1965@126.com; tianlei9010@126.com.
}

Background: Acute kidney injury (AKI), the most common complication after cardiac resuscitation, is highly prevalent and harmful. There is increasing evidence that levosimendan can improve cardiac output, increase renal blood flow, and prevent AKI. As a novel calcium sensitizer, levosimendan may exert its protective effect via mitochondria.

Methods: Rat models of asphyxia-induced cardiac arrest and cardiopulmonary resuscitation (CPR) were set up. Thirty healthy adult male SD rats were randomly divided into CPR group (CPR group, n=10), levosimendan-treated group (levo group, $\mathrm{n}=10$ ), and sham-operated group (sham group, $\mathrm{n}=10$ ). Twelve hours after CPR, serum renal function indicators were measured, the kidney injury and mitochondrial morphological changes were observed. Oxygen uptake of the mitochondria, mitochondrial adenosine triphosphate (ATP) and mitochondrial free $\mathrm{Ca}^{2+}$ concentration were measured. Oxidative stress-related indicator levels in rat kidney tissues were further detected to analyze the differences in apoptosis rates among these three groups. Mitochondrial optic atrophy 1 (Opa1), dynamin-related protein 1 (Drp1), and apoptosisrelated proteins were detected using Western blotting.

Results: Compared with the sham group, the CPR group had a significant increase in renal tissue damage. PAS staining and HE stains confirmed that CPR led to renal histopathological damage and destruction of the mitochondrial structure. Levosimendan improved the histopathological and ultrastructural damages of kidneys. Further analysis revealed that mitochondrial ATP content, NADH dehydrogenase, succinate dehydrogenase/cytochrome C oxidase, superoxide dismutase (SOD), catalase (CAT), and glutathione peroxidase $(\mathrm{CSH}-\mathrm{Px})$ decreased. Free $\mathrm{Ca}^{2+}$ concentration and malondialdehyde (MDA) significantly increased $($ all $\mathrm{P}<0.05)$ in the kidney tissues of rats in the CPR group. However, mitochondrial ATP content, NADH dehydrogenase, succinate dehydrogenase/cytochrome C oxidase, SOD, CAT, and CSH-Px increased, whereas free $\mathrm{Ca}^{2+}$ concentration and MDA decreased (all $\mathrm{P}<0.05$ ) in the levo group. The apoptosis rate increased in the CPR group. There were significantly increased levels of Drp1 protein levels, and significantly decreased Opal expression (all $\mathrm{P}<0.05$ ). However, the levo group showed the opposite effects (all $\mathrm{P}<0.05)$.

Conclusions: Levosimendan can alleviate AKI following CPR, which may be achieved by improving mitochondrial dysfunction and suppressing the mitochondrial apoptosis pathway.

Keywords: Cardiac arrest; cardiopulmonary resuscitation (CPR); acute kidney injury (AKI); levosimendan; mitochondria

Submitted Apr 02, 2021. Accepted for publication Jun 23, 2021.

doi: $10.21037 /$ tau-21-443

View this article at: https://dx.doi.org/10.21037/tau-21-443 


\section{Introduction}

The kidneys are the most vascularized organ in the body and one of the most common and earliest involved organs after cardiopulmonary resuscitation (CPR). Acute kidney injury (AKI) following CPR is strongly associated with systemic ischemia-reperfusion, with an incidence of $12-81 \%$. It increases the case-fatality rate, prolongs hospital stay, and is strongly associated with the shortand long-term prognosis of patients suffering from cardiac arrest $(1,2)$. Early identification and proper treatment of AKI following CPR can effectively improve outcomes from sudden cardiac arrest.

Mitochondria are an essential organelle that regulates cell function, differentiation, survival, and apoptosis. They are also one of the essential subcellular targets of ischemiareperfusion injury (3). The kidneys are one of the most oxygen-consuming organs, second only to the brain, and mitochondrial dysfunction is closely related to AKI (4). Adenosine triphosphate-sensitive potassium (KATP) channels have a role in regulating microcirculatory impairment and can improve ischemia-reperfusion injury (5). It has been proved that levosimendan, a non-selective KATP channel agonist, has a protective effect on AKI after cardiac damage (6). However, the specific mechanism via which levosimendan exerts such effect is still unclear. According to Aminzadeh et al. (7), levosimendan exerts its cardioprotective effect by inhibiting mitochondrial oxidative stress and alleviating mitochondrial dysfunction. Here we used rat models for cardiac arrest-CPR (CA/CPR) and investigated the effects of levosimendan on mitochondrial function in rats with AKI after CPR, mitochondrial energy metabolism, and mitochondrial kinetics as the primary measures. We present the following article in accordance with the ARRIVE reporting checklist (available at https://dx.doi.org/10.21037/ tau-21-443).

\section{Methods}

\section{Experimental animals}

Thirty specified pathogen-free (SPF) grade Sprague Dawley rats (weighing $510 \pm 6.6 \mathrm{~g}$ ) were provided by the Tang Wanchun Laboratories of Emergency Critical Care Medicine, Sun Yat-sen Memorial Hospital, Sun Yat-sen University. The animal production license number was SCXK (Guangdong) 2013-0034, and the animal use license number was SYXK (Guangdong) 2017-0081. Experiments were performed after a one-week adaptation. A protocol was prepared before the study without registration. Experiments were performed under the Ethics Committee of Renji Hospital, School of Medicine, Shanghai Jiao Tong University, in compliance with the institutional guidelines for the care and use of animals.

\section{Establishing the rat models of CA/CPR and animal grouping}

Rats were divided into the CPR group (CPR group, $\mathrm{n}=10$ ), levosimendan-treated group (levo group, $\mathrm{n}=10$ ), and shamoperated group (sham group, $\mathrm{n}=10$ ) randomly. The rats were fasted 12 hours before the experiment and anesthetized with $3 \%$ pentobarbital (Sigma-Aldrich, St. Louis, MO, USA) (45 mg/kg intraperitoneally and $10 \mathrm{mg} / \mathrm{kg} /$ hours intermittently intravenously during the operation). The right femoral artery and vein were cannulated for blood pressure measurement and intravenous drug administration. Electrocardiogram (ECG) monitoring was performed using a two-lead ECG recorder. The trachea of rats in the CPR group and levo group was blocked with a tracheostomy tube to create an asphyxiating environment. The heart rate and ECG changes were also monitored. Cardiac arrest was identified when the mean arterial pressure was below or equal to $20 \mathrm{mmHg}$, and the timer was reset for 6 minutes. At 5 minutes and 30 seconds of cardiac arrest, the rats were aspirated, followed by mechanical ventilation with $100 \%$ oxygen at a rate of 200 breaths/minute. At 6 minutes of cardiac arrest, external chest compressions were applied $2.8 \mathrm{~cm}$ above the xiphoid process at a rate of 200 compressions/minutes and a compression depth of $1 \mathrm{~cm}$. Coronary perfusion pressure was supported at $22 \pm 2 \mathrm{mmHg}$ during the compressions. After resuscitation, the mean arterial pressure reached $60 \mathrm{mmHg}$ or more for 5 minutes was considered the return of spontaneous circulation. Failure to restore normal cardiac rhythm after 20 minutes of continuous chest compressions was considered failed resuscitation. Rats in the sham group were intubated but did not undergo CPR, and the relevant indicators were also recorded. In the levo group, levosimendan $12 \mu \mathrm{g} / \mathrm{kg}$ (Qilu Pharmaceutical Co. Ltd, Shandong, China) was administered intravenously at 5 minutes $30 \mathrm{sec}$ of cardiac arrest, and a maintenance dose $(0.3 \mu \mathrm{g} / \mathrm{kg} /$ minutes $)$ was applied after resuscitation; in the CPR and sham groups, equal doses of normal saline were injected simultaneously.

\section{Detection of renal function indicators}

Blood samples were collected from the femoral vein of these rats 12 hours after CPR and centrifuged at 3,000 rpm 
for 10 minutes to harvest the serum. The serum creatinine (Scr), blood urea nitrogen (Bun), and blood creatinine were measured by ELISA kit (Nanjing Jiangcheng Bioengineering Institute, Nanjing, China) according to the instructions provided. Before the rats were sacrificed, 24-hour urine samples were collected from each group and centrifuged at 3,500 rpm for 10 minutes to remove the precipitate. Urine $\beta 2$ microglobulin ( $\beta 2-M G)$ and neutrophil gelatinase-associated lipocalin (NGAL) levels were measured using a commercial ELISA kit (Nanjing Jiangcheng Bioengineering Institute). Optical density (OD) values were measured at $450 \mathrm{~nm}$ using a microplate reader (BioTek Instruments Inc., VT, USA). Standard curves were created with the concentration and the corresponding OD value.

\section{Histopathological examinations of the kidneys}

Twelve hours after CPR, the rats were anesthetized with phenobarbital sodium [intraperitoneal (i.p.)]. The left kidney was harvested and rinsed well with pre-chilled saline to remove blood stains. The kidney tissues fixed in the $10 \%$ formaldehyde solution were conventionally dehydrated, embedded in paraffin, and cut into 5 - $\mu \mathrm{m}$ thick serial sections. After dewaxing with xylene, the sections were immersed in hematoxylin for staining for several minutes, followed by dehydration in ethanol. After staining with an eosin solution (Reagent No. 3 Factory of Shanghai Chemical Reagent, Shanghai, China) for 2-3 minutes, the sections were routinely dehydrated, made transparent, and sealed. The histopathological changes in the kidneys were observed under Olympus BX50 light microscope (Olympus, Tokyo, Japan), and the Image ProPlus software was applied to score the pathological changes, including structural destruction and cast formation in renal tubules.

Subsequently, the dewaxed sections were oxidized using periodate solution (Reagent No. 3 Factory of Shanghai Chemical Reagent) for 10 minutes, washed with water, and stained with Schiff solution for 10-15 minutes. After nuclear staining with hematoxylin for 1-2 minutes and washed with running water, the sections were differentiated using $1 \%$ hydrochloric ethanol for 20 seconds and washed with warm water for 5-10 minutes until turning the sky blue. Finally, the sections were observed under a light microscope after ethanol dehydration, xylene transparency, and neutral gum mounting.

\section{Electron microscopy of changes in mitochondrial morphology}

Renal tissue samples were harvested. A 1- $\mathrm{mm}^{3}$ renal cortex tissue was taken and fixed with $2.5 \%$ glutaraldehyde (Ruinan Biotechnology Center, Shanghai, China) within 1 h. $1 \mathrm{~mm}^{3}$ of renal tissue was obtained from each animal within 1 hour, including a portion of the renal cortex, and fixed in $2.5 \%$ glutaraldehyde (Ruinan Biotechnology Center, Shanghai, China). The tissues were then rinsed, fixed in $1 \%$ osmium tetroxide solution (Ruinan Biotechnology Center), and dehydrated in an ethanol-acetone series. The sections were then placed in propylene oxide and embedded before they were cut in ultrathin $(50 \mathrm{~nm})$ sections, which were doublestained with uranyl acetate and lead citrate, and examined with a JEM-1210 electron microscope (JEOL Ltd., Tokyo, Japan).

\section{Determination of mitochondrial respiratory enzyme activities, ATP content, and free calcium $\left(\mathrm{Ca}^{2+}\right)$ level}

The kidney tissues of rats in each group were weighed and homogenized by adding Tris- $\mathrm{HCl}$ buffer in weight to volume ratio of $1: 5$ to make a $10 \%$ tissue homogenate, which was centrifuged twice at 2,000 rpm for 15 minutes at low temperature to remove the precipitate. The supernatants obtained were mixed and centrifuged twice at $12,000 \mathrm{rpm}$ for 15 minutes at low temperature. The precipitate was discarded, and the remaining suspension was used as mitochondrial preparation for the experiments. The activities of the mitochondrial respiratory enzymes, including NADH dehydrogenase, succinate dehydrogenase, and cytochrome $\mathrm{C}$ oxidase, were measured using a modified Clark-type electrode. After ultrasonically treating the mitochondria, colorimetric assay determined the ATP content using a UV-Vis spectrophotometer. Finally, free calcium $\left(\mathrm{Ca}^{2+}\right)$ was determined with calcium carbonate by using a chemiluminescence assay.

\section{Detection of oxidative stress indicators in kidney tissues}

The kidney tissues were rinsed with pre-cooled normal saline, dried with filter paper, and weighed. Then, $200 \mathrm{mg}$ of kidney tissue was cut up and ground on ice with the required volume of normal saline. After it was centrifuged at $3,000 \mathrm{rpm}$ for 10 minutes, the supernatant was obtained and reacted with an ELISA kit (Nanjing Jiangcheng Bioengineering Institute) at room temperature for 30 minutes. The $\mathrm{OD}$ values were read on a plate reader. The levels of superoxide dismutase (SOD), catalase (CAT), glutathione peroxidase (CSH-Px), and malondialdehyde (MDA) were calculated according to the standard curve. 
Table 1 Changes in biochemical indicators of blood and urine in each group

\begin{tabular}{lccccc}
\hline Group & $\mathrm{n}$ & $\operatorname{Scr}(\mu \mathrm{mol} / \mathrm{L})$ & Bun $(\mathrm{mmol} / \mathrm{L})$ & $\beta 2-\mathrm{MG}(\mathrm{mg} / \mathrm{L})$ & $\mathrm{NGAL}(\mathrm{ng} / \mathrm{mL})$ \\
\hline Sham group & 10 & $16.89 \pm 4.035$ & $2.776 \pm 0.4936$ & $0.083 \pm 0.021$ & $7.089 \pm 2.931$ \\
CPR group & 10 & $81.81 \pm 7.854^{\mathrm{a}}$ & $6.647 \pm 1.161^{\mathrm{a}}$ & $0.155 \pm 0.064^{\mathrm{a}}$ & $8.425 \pm 4.270^{\mathrm{a}}$ \\
Levo group & 10 & $33.25 \pm 3.278^{\mathrm{a}, \mathrm{b}}$ & $4.541 \pm 1.129^{\mathrm{a}, \mathrm{b}}$ & $0.117 \pm 0.032^{\mathrm{a}, \mathrm{b}}$ & $7.618 \pm 3.364^{\mathrm{a},}$ \\
\hline
\end{tabular}

${ }^{a}, \mathrm{P}<0.05$, compared with the sham group; ${ }^{b}, \mathrm{P}<0.05$, compared with the CPR group. CPR, cardiopulmonary resuscitation.

\section{Identification of apoptosis in kidney tissue sections}

The paraffin-embedded sections were deparaffinized, rehydrated sequentially in xylene and gradient ethanol, and then washed with PBS twice (5 min each). After the water was removed, proteinase $\mathrm{K}$ was added dropwise for digestion at $37{ }^{\circ} \mathrm{C}$ for $10-30$ minutes. After the slides were washed with PBS, the TUNEL solution (Promega, Madison, USA) was prepared and incubated with the sample at $37^{\circ} \mathrm{C}$ for 60 minutes in the dark. After washing with PBS 3 times, DAPI (Beyotime Biotechnology, Shanghai, China) was added dropwise for incubation at $37{ }^{\circ} \mathrm{C}$ for 10 minutes before the mixture was sealed with a coverslip. Under an Olympus fluorescence microscope (Olympus, Tokyo, Japan), five regions (200x) were randomly selected from each section for analysis. The percentage of TdT-mediated dUTP-biotin nick end labeling (TUNEL)-positive cells among 4'-6-diamidino-2-phenylindole (DAPI)-positive cells was calculated, and the average value from these sections was taken as the apoptosis rate.

\section{Western blotting}

The levels of mitochondrial optic atrophy 1 (Opa1), dynamin-cleaved caspase 1 (Drp1), cytochrome C (Cyt C), cleaved caspase-9, and cleaved caspase- 3 were detected by using Western blotting. Precisely, $100 \mathrm{mg}$ of rat kidney tissue was cut into pieces, homogenized, and centrifuged at $12,000 \mathrm{rpm}$ for $5 \mathrm{~min}$. Protein was extracted and measured with BCA assay. For each group, $50 \mu \mathrm{g}$ of protein was electrophoresed on sodium dodecyl sulfate-polyacrylamide gel electrophoresis (SDS-PAGE) gel and electrotransferred polyvinyl fluoride membranes. After having been sealed at room temperature for 1 hour, the membranes were probed with primary rabbit anti-rat antibodies against cleaved caspase-3 (1:500, Cell Signaling Technology Inc., MA, USA), cleaved caspase-9 (1:500, Cell Signaling Technology Inc.), Bax (1:500, Cell Signaling Technology Inc.), Bcl-2 (1:500, Cell Signaling Technology Inc.), Cyt C (1:500, Cell
Signaling Technology Inc.), Dpr1 (1:1,000, Proteintech Group, Chicago, USA), Opa1 (1:1,000, Proteintech Group), and GAPDH (1:1,000, Proteintech Group), respectively, and then incubated overnight at $4{ }^{\circ} \mathrm{C}$. The membranes were washed using TBST and incubated with horseradish peroxidase-conjugated goat anti-rabbit secondary antibody $(1: 5,000$, Beyotime Biotechnology). The membranes were washed using TBST three times ( 5 minutes each time), and then the signals were detected using an electrochemiluminescence reagent. Image J software was used to analyze the Western blotting data, with GAPDH used as the internal reference.

\section{Statistical analysis}

Statistical analysis was performed using GraphPad Prism 7.0 software. All the typically distributed measures are presented as $\bar{x} \pm$ SD. Independent samples $t$-test was used to compare the difference between two groups, and one-way analysis of variance (ANOVA) for multiple groups, with a $\mathrm{P}$ value of $<0.05$ being considered statistically significant.

\section{Results}

Effect of levosimendan on kidney function in rats with AKI following CPR

Compared with the sham group, the CPR group had significantly higher levels of 12-hour Scr and Bun after CPR, along with significantly higher levels of urinary $\beta 2$ $M G$ and NGAL $(P<0.05)$; in the levo group, however, the levels of Scr, Bun, $\beta 2-M G$, and NGAL significantly decreased $(\mathrm{P}<0.05)($ Table 1).

\section{Effect of levosimendan on renal histopathological damage in rats with AKI following CPR}

Hematoxylin-eosin (HE) staining showed that the renal tissue of rats in the sham group had typical structures, with 

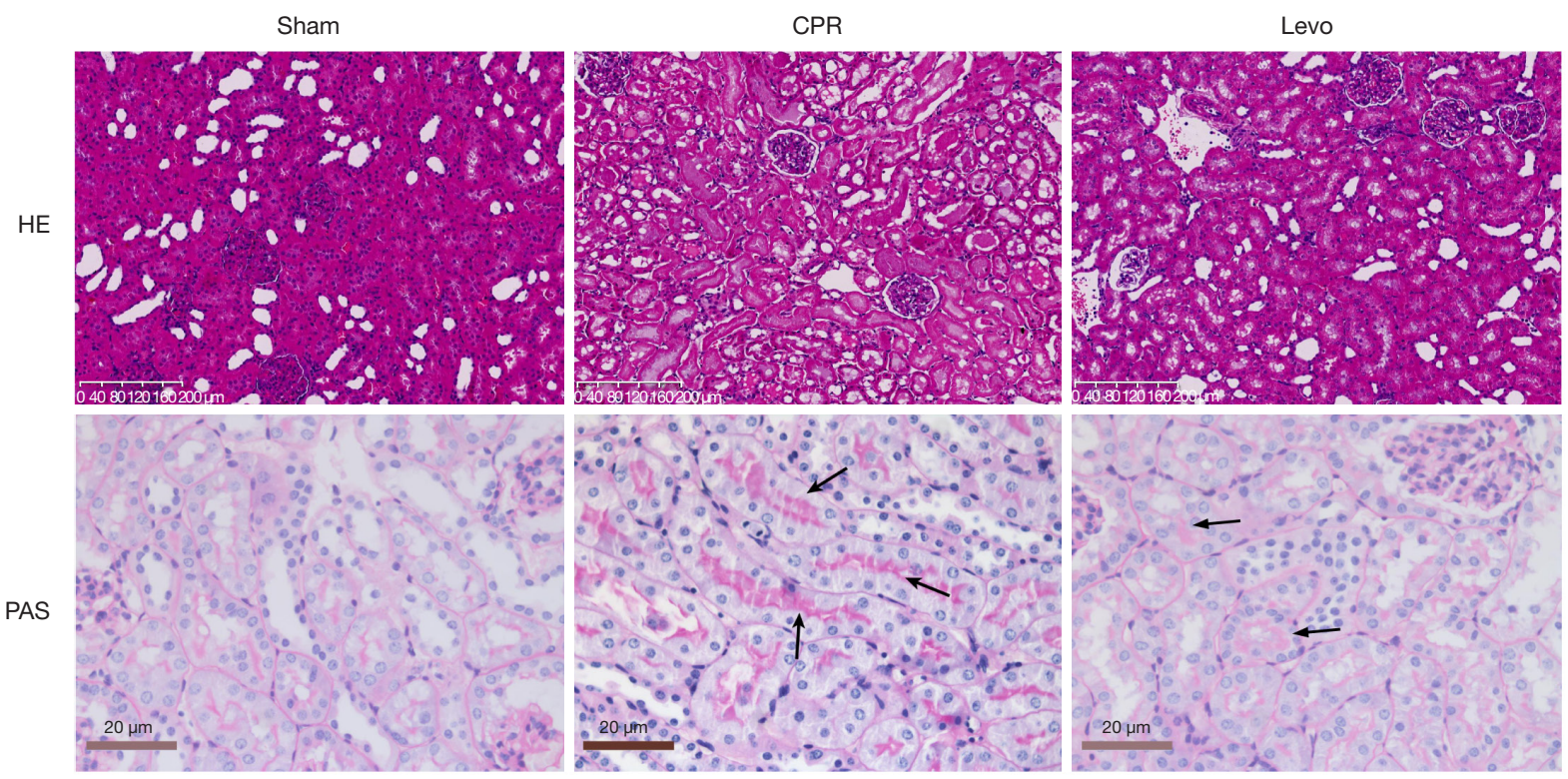

Figure $1 \mathrm{HE}$ stains and PAS staining of kidney tissues in each group (200x magnification). The black arrows show the red-stained casts. CPR, cardiopulmonary resuscitation; PAS, periodic acid-Schiff.

the infiltration of only a limited number of inflammatory cells. However, rats in the CPR group showed apparent structural destruction of renal tubules, including the detachment of brush border in the lumen, apparent shrinking of Bowman space, and more inflammatory cells in the tiny vessels. In the levo group, cell swelling, death, and detachment still existed in renal glomeruli and tubules; however, how much damage was less than that in the CPR group. The results of periodic acid-Schiff (PAS) staining showed that: the structures of renal tissue were typical in the sham group, with only a tiny amount of red-stained structures; in the CPR group, the lumen of the renal tubules was dilated, in which many homogeneously red-stained casts were visible, along with the degeneration and swelling of the renal tubular epithelial cells; however, the casts in the lumen were significantly reduced in the levo group compared with the CPR group, along with remarkably alleviated lesions (Figure 1).

\section{Effect of levosimendan on renal mitochondrial morphology in rats with $A K I$ following $C P R$}

Under an electron microscope, mitochondria in the sham group had an intact structure and normal morphology. In the CPR group, the number of mitochondria decreased, some mitochondria's size became larger and rounder, with reduced and broken cristae, unclear structure, and disorganized arrangement. In the levo group, the mitochondrial structure was remarkably improved. How much swelling was notably attenuated, the number of cristae was typical, and the arrangement became orderly (Figure 2).

\section{Effect of levosimendan on mitochondrial respiratory enzyme activities in rats with AKI following CPR}

As shown in Table 2, compared with the sham group, the CPR group had significantly lower levels of mitochondrial NADH dehydrogenase, succinate dehydrogenase, and cytochrome $\mathrm{C}$ oxidase in the kidney tissues (all $\mathrm{P}<0.05$ ); compared with the CPR group, the levo group had significantly increased activities of these mitochondrial respiratory enzymes (all $\mathrm{P}<0.05)$.

\section{Effect of levosimendan on mitochondrial energy metabolism in renal tissues in rats with AKI following CPR}

Compared with the sham group, the mitochondrial ATP content in renal tissues was significantly lower, and the free $\mathrm{Ca}^{2+}$ concentration was significantly higher in the CPR group (both $\mathrm{P}<0.05$ ); compared with the $\mathrm{CPR}$ group, the mitochondrial ATP content was significantly higher, and 

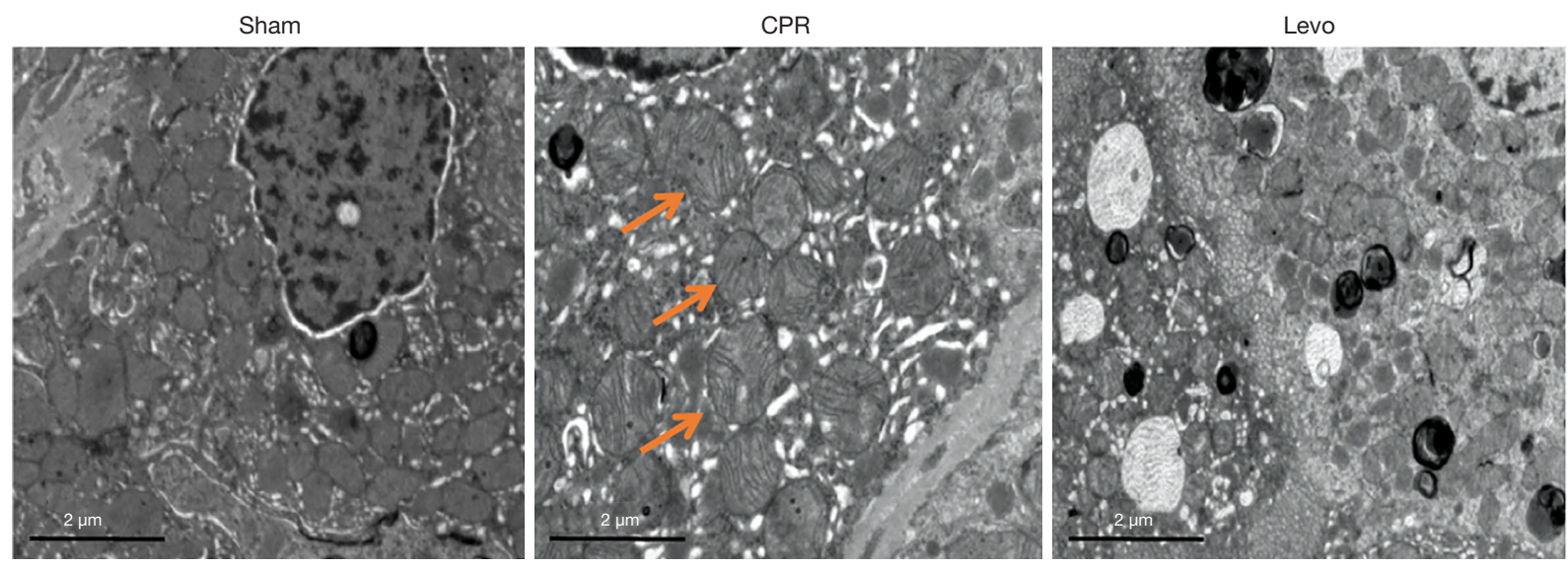

Figure 2 Mitochondrial structures in kidney tissues in each group. The yellow arrows show the swollen mitochondria. CPR, cardiopulmonary resuscitation.

Table 2 Mitochondrial respiratory enzyme activities in each group

\begin{tabular}{lcccc}
\hline Group & $\mathrm{n}$ & $\begin{array}{c}\mathrm{NADH} \text { dehydrogenase } \\
{[\mathrm{nmol} /(\text { minutes } \times \mathrm{mg})]}\end{array}$ & $\begin{array}{c}\text { Succinate dehydrogenase } \\
{[\mathrm{nmol} /(\text { minutes } \times \mathrm{mg})]}\end{array}$ & $\begin{array}{c}\text { Cytochrome c oxidase } \\
{[\mathrm{nmol} /(\mathrm{minutes} \times \mathrm{mg})]}\end{array}$ \\
\hline Sham group & 10 & $547 \pm 51.79$ & $371.2 \pm 35.17$ & $850 \pm 47.94$ \\
CPR group & 10 & $245.7 \pm 35.23^{\mathrm{a}}$ & $109.1 \pm 14.43^{\mathrm{a}}$ & $426 \pm 39.40^{\mathrm{a}}$ \\
Levo group & 10 & $398 \pm 54.25^{\mathrm{a}, \mathrm{b}}$ & $176.5 \pm 25.58^{\mathrm{a}, \mathrm{b}}$ & $621.7 \pm 34.85^{\mathrm{a}, \mathrm{b}}$ \\
\hline
\end{tabular}

${ }^{a}, \mathrm{P}<0.05$, compared with the sham group; ${ }^{\mathrm{b}}, \mathrm{P}<0.05$, compared with the CPR group. CPR, cardiopulmonary resuscitation.

Table 3 Mitochondrial ATP content and free $\mathrm{Ca}^{2+}$ concentration in kidney tissues in each group

\begin{tabular}{lccc}
\hline Group & $\mathrm{n}$ & ATP $[\mu \mathrm{mol} /(\mathrm{g} \times \mathrm{pro})]$ & Free Ca $^{2+}[\mathrm{nmol} /(\mathrm{g} \times \mathrm{pro})]$ \\
\hline Sham group & 10 & $0.9630 \pm 0.04206$ & $11.28 \pm 1.026$ \\
CPR group & 10 & $0.4460 \pm 0.02296^{\mathrm{a}}$ & $31.00 \pm 2.145^{\mathrm{a}}$ \\
Levo group & 10 & $0.6380 \pm 0.03190^{\mathrm{a}, \mathrm{b}}$ & $19.34 \pm 1.301^{\mathrm{a}, \mathrm{b}}$ \\
\hline
\end{tabular}

${ }^{\mathrm{a}}, \mathrm{P}<0.05$, compared with the sham group; ${ }^{\mathrm{b}}, \mathrm{P}<0.05$, compared with the CPR group. ATP, adenosine triphosphate; CPR, cardiopulmonary resuscitation.

the free $\mathrm{Ca}^{2+}$ concentration was significantly lower (both $\mathrm{P}<0.05$ ) in the levo group (Table 3).

\section{Effect of levosimendan on renal oxidative stress in rats with AKI}

Compared with the sham group, the levels of SOD, CSH-Px, and CAT in the kidney tissues of rats in the CPR group were significantly decreased, and the level of MDA was significantly increased (all $\mathrm{P}<0.05$ ). The levels of SOD, CSH-Px, and CAT in the kidney tissues of rats in the levo group were significantly increased compared with the CPR group, while the level of
MDA significantly increased (all $\mathrm{P}<0.05)$ (Figure 3).

\section{Effect of levosimendan on renal cell apoptosis in rats with AKI}

The results of the TUNEL assay showed that the rats in the sham group showed very weak green fluorescence and a low apoptosis rate. However, the CPR group had significantly more green fluorescent particles and enhanced fluorescence intensity, and the quantitative analysis suggested an increased apoptosis rate $(\mathrm{P}<0.05)$. The levo group had significantly fewer green fluorescent particles 

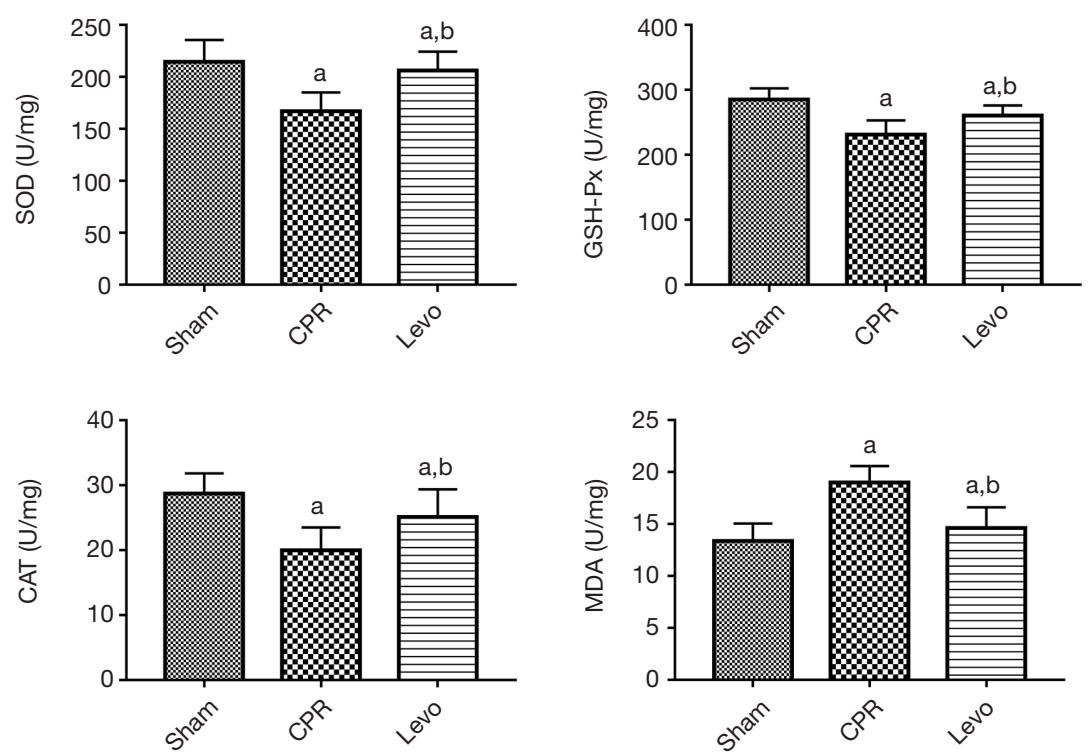

Figure 3 Change of oxidative stress indicators in kidney tissues in each group. a, $\mathrm{P}<0.05$, compared with the sham group; $\mathrm{b}$, $\mathrm{P}<0.05$, compared with the CPR group. CPR, cardiopulmonary resuscitation.

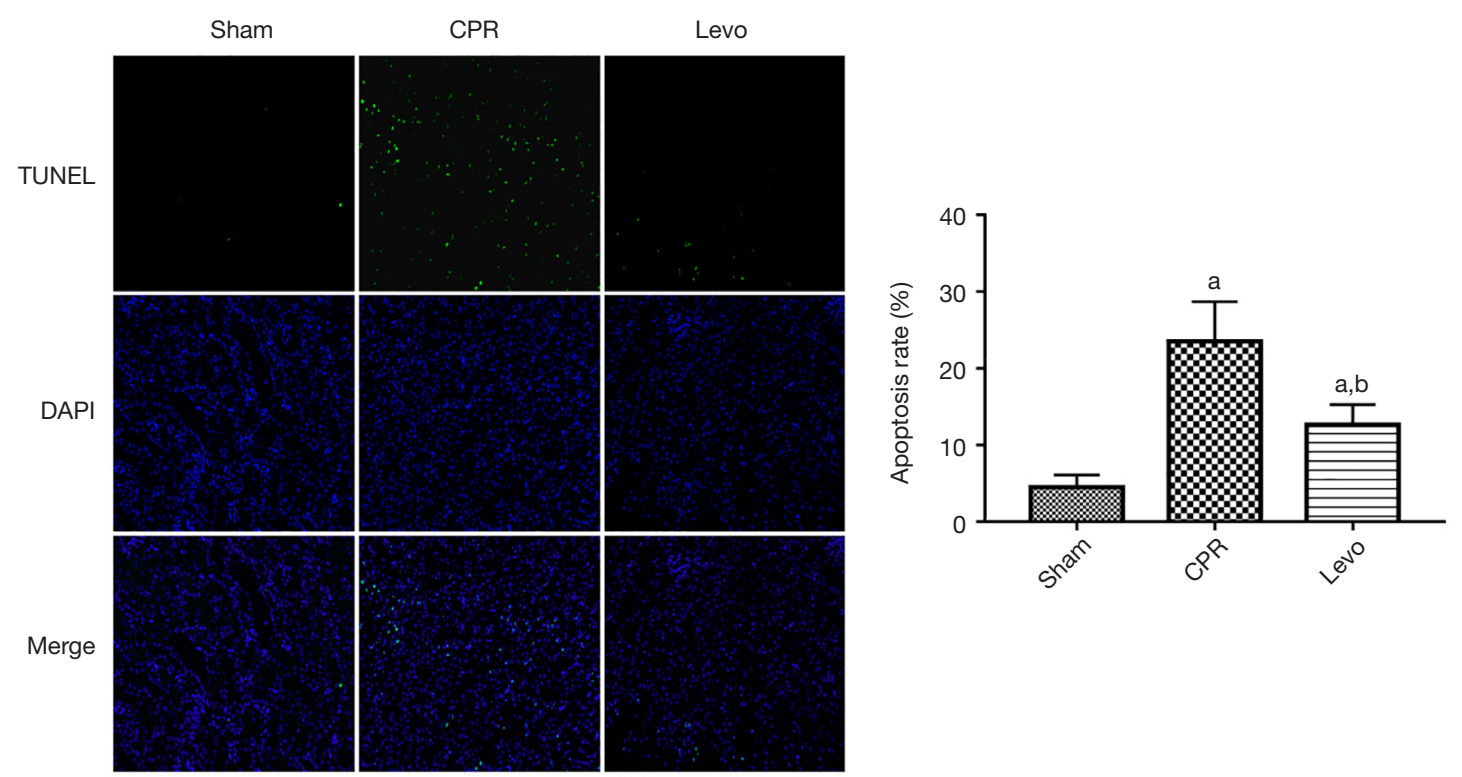

Figure 4 TUNEL assay of apoptosis of renal cells in each group (200× magnification). a, $\mathrm{P}<0.05$, compared with the sham group; b, $\mathrm{P}<0.05$, compared with the CPR group. CPR, cardiopulmonary resuscitation.

and a significantly lower apoptosis rate than the $\mathrm{CPR}$ group $(\mathrm{P}<0.05)$ (Figure 4).

\section{Effect of levosimendan on mitochondrial fusion and fission in renal tissues in rats with AKI following CPR}

Western blotting results showed that, compared with the sham group, the CPR group had significantly higher expression of Drp1, which promotes mitochondrial fission in renal tissue, and significantly lower expression of mitochondrial fusion protein Opa1 $(\mathrm{P}<0.05)$; compared with the CPR group, the expression of Drp1 decreased significantly while the expression of Opa1 increased significantly $(\mathrm{P}<0.05)$ in the levo group (Figure 5). 

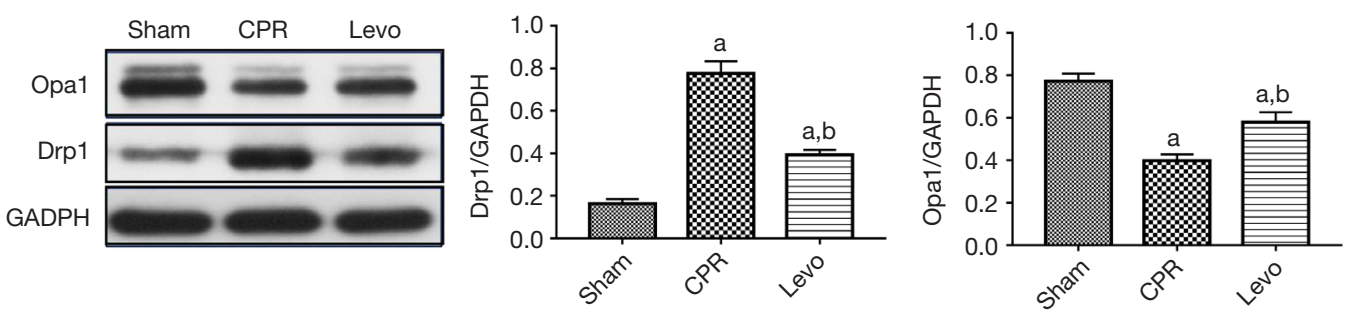

Figure 5 Expression levels of Drp1 and Opa1 in kidney tissues in each group. a, $\mathrm{P}<0.05$, compared with the sham group; b, $\mathrm{P}<0.05$, compared with the CPR group. CPR, cardiopulmonary resuscitation.
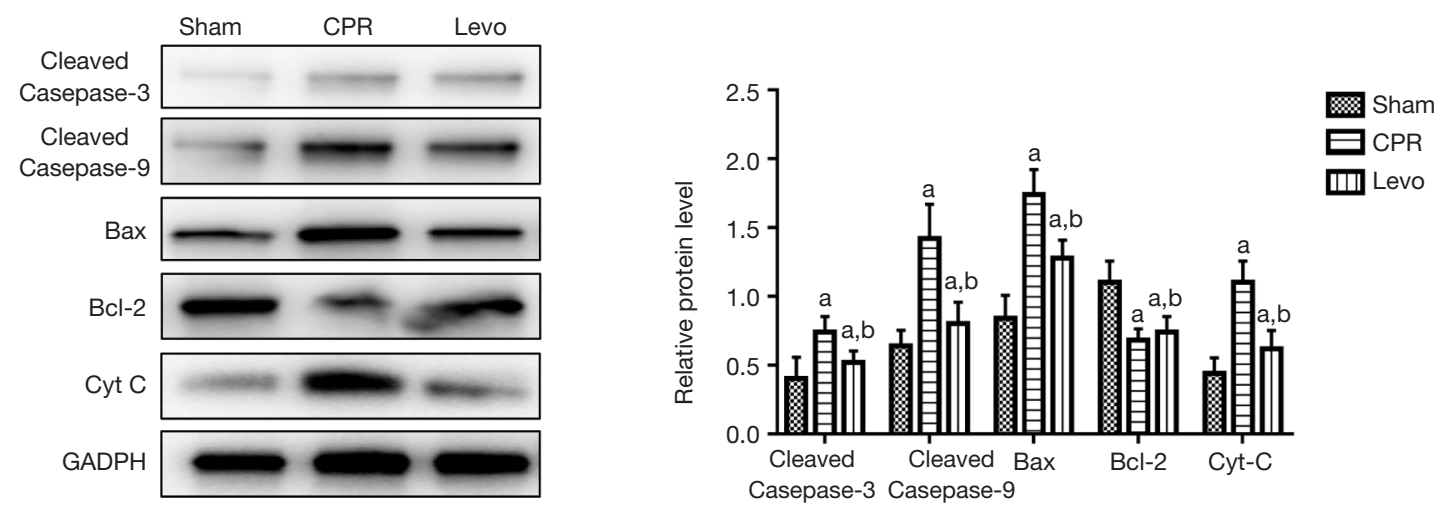

Figure 6 Expression levels of Cyt $\mathrm{C}$ and caspase proteins in kidney tissues in each group. a, $\mathrm{P}<0.05$, compared with the sham group; $\mathrm{b}$, $\mathrm{P}<0.05$, compared with the $\mathrm{CPR}$ group. $\mathrm{CPR}$, cardiopulmonary resuscitation.

\section{Effect of levosimendan on Cyt C and caspases}

Western blotting revealed that, compared with the sham group, the CPR group had significantly up-regulated Bax expression and down-regulated Bcl-2 expression, which promoted Cyt $\mathrm{C}$ release, resulting in higher levels of cleaved caspase- 9 and cleaved caspase- $3(\mathrm{P}<0.05)$. In the levo group, the content of Bax in kidney tissues was significantly reduced, the content of $\mathrm{Bcl}-2$ was significantly increased, and the expressions of Cyt C, cleaved caspase-9, and cleaved caspase- 3 were decreased to different degrees $(\mathrm{P}<0.05)$ (Figure 6).

\section{Discussion}

Ischemia-reperfusion injury is the primary pathological mechanism for AKI development after CPR (8), and levosimendan can improve ischemia-reperfusion injury in tissues and organs. Mitochondrial dysfunction plays a vital role in the development of renal diseases (9). One of our earlier studies has confirmed that levosimendan could improve AKI after CPR (6), but the specific mechanism was not elucidated. In our current study, levosimendan alleviating AKI after CPR in rats by enhancing mitochondrial respiratory enzyme activities, promoting mitochondrial energy metabolism, regulating mitochondrial kinetics-related protein expressions, improving mitochondrial dysfunction, and reducing the number of apoptotic cells caused by the mitochondrial pathway. Additionally, the side effects and potential risks of levosimendan are relatively small, which was also indicated in our previous study (10).

As key sites of cellular respiration and energy metabolism, mitochondria play a significant role in various cellular physiological processes. The kidney is the second most mitochondria-rich organ, and the renal tubules are the most mitochondria-dense parts in nephrons (11). Mitochondria are a critical contributor to the pathogenesis of AKI. Many mitochondria-related factors, including mitochondrial energy metabolism, mitochondrial kinetics, oxidative stress, mitochondrial autophagy, and mitochondrial biosynthesis, participate in regulating the development of AKI $(12,13)$. 
ATP production through the respiratory chain reaction is an essential process of mitochondrial energy metabolism, and abnormal energy metabolism due to mitochondrial dysfunction is crucial to ischemia-hypoxia-reperfusion injury in the kidney (14). A recent study (15) reported that depletion of ATP in the mitochondria of proximal tubule (PT) cells plays a significant role in the pathogenesis of AKI and is associated with long-term renal fibrosis. In our current study, mitochondrial respiratory enzyme activities were significantly reduced in rat kidneys after CPR, decreased ATP synthesis, and increased free $\mathrm{Ca}^{2+}$ concentration. Normal mitochondrial uptake may help support normal calcium homeostasis. Simultaneously, impaired ATP synthesis will cause calcium overload, which inhibits oxidative phosphorylation and further suppresses ATP synthesis, forming a vicious cycle of "impaired ATP synthesis-calcium overload-impaired ATP synthesis" and ultimately aggravating the renal injury. In our current study, levosimendan was found to increase mitochondrial respiratory enzyme activities and ATP content and decrease mitochondrial free $\mathrm{Ca}^{2+}$ level, suggesting that levosimendan can improve mitochondrial energy metabolism in kidneys.

Mitochondria are highly dynamic organelles undergoing coordinated cycles of fission and fusion to support normal cellular functions. Mitochondrial dynamics are altered during AKI, leading to mitochondrial fragmentation and promoting damage and apoptosis of renal tubular cells (16-18). Impaired mitochondrial fusion is a crucial contributor to mitochondrial fragmentation. In AMI models with comorbid sepsis, the decreased Opa1 protein expression promoted mitochondrial fragmentation and aggravated renal injury (19). Liu et al. (20) found mitochondrial Drp1 protein levels increased, and mitochondrial fragmentation was enhanced in kidney tissues during sepsis-induced AKI. In our current study, Drp1 expression increased, and Opa1 expression decreased during AKI following CPR; however, levosimendan intervention decreased Drp1 expression and increased Opa1 expression. Thus, levosimendan may be involved in regulating mitochondrial dynamics.

Recent studies have shown that impaired mitochondrial respiratory chain complexes caused by ischemia-reperfusion produce excessive ROS, which damages proteins, lipids, and nucleic acids in surrounding normal tissues and activates a series of molecular signals (21). Cells have formed a complex antioxidant defense system, including SOD, MDA, and GSH, to protect the organism from peroxidative damage (22). SOD converts superoxide anions into hydrogen peroxide, and CAT, in turn, converts hydrogen peroxide into harmless water molecules. GSH, when catalyzed, is converted to oxidized glutathione, which reduces the oxidized substance and relieves its toxicity (23). In our current study, levosimendan inhibited the increase of MDA caused by CPR and increased the SOD, CAT, and GSH-Px levels. These findings suggest levosimendan has a protective effect on AKI caused by CPR. These results may be related to the effects of levosimendan in enhancing the body's antioxidant system, inhibiting peroxidative lipid reactions, and scavenging ROS.

Mitochondria are the energy center of cells and central sites for regulating cellular apoptosis. There is a high apoptosis level during AKI. Tonnus et al. (24) concluded apoptosis is a crucial feature of renal tubular cell death after AKI. In our current study, TUNEL staining revealed that CPR could significantly increase the number of apoptotic cells. However, the number of apoptotic cells was significantly lowered after levosimendan treatment, suggesting levosimendan could inhibit the apoptosis of renal cells. In ischemia-reperfusion, massive ROS formation and $\mathrm{Ca}^{2+}$ overload will trigger mitochondrial swelling, decrease in membrane potential, and opening of supramembrane channels.

Meanwhile, pro-apoptotic factors, including Cyt $\mathrm{C}$ in mitochondria, are released from the damaged mitochondrial outer membrane to the cytosol and then bound to apoptotic protease activating factor 1 (Apaf-1) to recruit and activate caspase-9/3, which starts the caspase cascade that worsens cell injury. As a critical molecule downstream of the mitochondrial apoptotic pathway, the cleaved caspase- 3 can reflect the level of apoptosis (25-27). In the present study, Western blotting showed that CPR elevated the expressions of Cyt C, cleaved caspase-9, and cleaved caspase-3 proteins, suggesting CPR can trigger apoptosis in the mitochondrial pathway while levosimendan can suppress such apoptosis. The Bcl-2 family is a crucial regulator of the apoptotic pathway, of which Bcl-2 and Bax are the prominent antiapoptotic and pro-apoptotic members, respectively (28). $\mathrm{Bcl}-2$ proteins are distributed in the mitochondrial outer membrane, endoplasmic reticulum, and perinuclear membrane and inhibit apoptosis by suppressing the decrease in mitochondrial membrane potential and inhibiting the release of apoptosis-inducing factor (AIF) and Cyt C (29). However, Bax is present in the cytoplasm and induces apoptosis (30). In the present study, we found a significant decrease in Bax expression and a significant increase in Bcl-2 expression in the renal tissues of rats in the levo group, again suggesting that levosimendan inhibits apoptosis 
of renal cells. Moreover, several studies reported that levosimendan has anti-inflammatory, antioxidant and antiapoptotic effects, which may be one of the ways to exert its renal protective effect, but further research is needed.

The shortcomings of this study are as follows: (I) we only analyzed the role of mitochondria in levosimendan alleviating acute kidney injury, and did not analyze other related mechanisms; (II) due to the high mortality after $12 \mathrm{~h}$ of CPR modeling, we did not conduct multiple time points detection. Follow-up studies would be required to confirm it.

In conclusion, levosimendan alleviates AKI after CPR in rats by enhancing mitochondrial respiratory enzyme activities, promoting mitochondrial energy metabolism, regulating mitochondrial kinetics-related protein expressions, alleviating mitochondrial dysfunction and oxidative stress, and suppressing apoptosis.

\section{Acknowledgments}

Funding: This work was supported by the National Natural Science Foundation of China general program (81971803) and Shanghai Municipal Health Commission Research Project (20184Y0126).

\section{Footnote}

Reporting Checklist: The authors have completed the ARRIVE reporting checklist. Available at https://dx.doi. org/10.21037/tau-21-443

Data Sharing Statement: Available at https://dx.doi. org/10.21037/tau-21-443

Conflicts of Interest: All authors have completed the ICMJE uniform disclosure form (available at https://dx.doi. org/10.21037/tau-21-443). The authors have no conflicts of interest to declare.

Ethical Statement: The authors are accountable for all aspects of the work in ensuring that questions related to the accuracy or integrity of any part of the work are appropriately investigated and resolved. Experiments were performed under the Ethics Committee of Renji Hospital, School of Medicine, Shanghai Jiao Tong University, in compliance with the institutional guidelines for the care and use of animals.

Open Access Statement: This is an Open Access article distributed in accordance with the Creative Commons Attribution-NonCommercial-NoDerivs 4.0 International License (CC BY-NC-ND 4.0), which permits the noncommercial replication and distribution of the article with the strict proviso that no changes or edits are made and the original work is properly cited (including links to both the formal publication through the relevant DOI and the license). See: https://creativecommons.org/licenses/by-nc-nd/4.0/.

\section{References}

1. Dutta A, Hari KJ, Azizian J, et al. Incidence, Predictors, and Prognosis of Acute Kidney Injury Among Cardiac Arrest Survivors. J Intensive Care Med 2021;36:550-6.

2. Mah KE, Alten JA, Cornell TT, et al. Acute kidney injury after in-hospital cardiac arrest. Resuscitation 2021;160:49-58.

3. Tammaro A, Kers J, Scantlebery AML, et al. Metabolic Flexibility and Innate Immunity in Renal Ischemia Reperfusion Injury: The Fine Balance Between Adaptive Repair and Tissue Degeneration. Front Immunol 2020;11:1346.

4. An S, Luo H, Wang J, et al. An acute kidney injury prediction nomogram based on neurosurgical intensive care unit profiles. Ann Transl Med 2020;8:194.

5. Pertiwi KR, Hillman RM, Scott CA, et al. Ischemia Reperfusion Injury Produces, and Ischemic Preconditioning Prevents, Rat Cardiac Fibroblast Differentiation: Role of KATP Channels. J Cardiovasc Dev Dis 2019;6:22.

6. Thorlacius EM, Suominen PK, Wåhlander H, et al. The Effect of Levosimendan Versus Milrinone on the Occurrence Rate of Acute Kidney Injury Following Congenital Heart Surgery in Infants: A Randomized Clinical Trial. Pediatr Crit Care Med 2019;20:947-56.

7. Aminzadeh A, Mehrzadi S. Cardioprotective effect of levosimendan against homocysteine-induced mitochondrial stress and apoptotic cell death in H9C2. Biochem Biophys Res Commun 2018;507:395-9.

8. Fu ZY, Wu ZJ, Zheng JH, et al. The incidence of acute kidney injury following cardiac arrest and cardiopulmonary resuscitation in a rat model. Ren Fail 2019;41:278-83.

9. Kislitsina ON, Rich JD, Wilcox JE, et al. Shock Classification and Pathophysiological Principles of Therapeutics. Curr Cardiol Rev 2019;15:102-13.

10. Jia T, Wang S, Luo C, et al. Levosimendan Ameliorates Post-Resuscitation Acute Intestinal Microcirculation Dysfunction Partly Independent of Its Effects on Systemic Circulation: A Pilot Study On Cardiac Arrest In A Rat 
Model. Shock 2021. [Epub ahead of print]. doi: 10.1097/ SHK.0000000000001771.

11. Arulkumaran N, Pollen S, Greco E, et al. Renal Tubular Cell Mitochondrial Dysfunction Occurs Despite Preserved Renal Oxygen Delivery in Experimental Septic Acute Kidney Injury. Crit Care Med 2018;46:e318-25.

12. Sun J, Zhang J, Tian J, et al. Mitochondria in SepsisInduced AKI. J Am Soc Nephrol 2019;30:1151-61.

13. Qi J, Xue Q, Kuang L, et al. Berberine alleviates cisplatininduced acute kidney injury by regulating mitophagy via PINK 1/Parkin pathway. Transl Androl Urol 2020;9:1712-24.

14. Bhargava P, Schnellmann RG. Mitochondrial energetics in the kidney. Nat Rev Nephrol 2017;13:629-46.

15. Yamamoto $S$, Yamamoto $M$, Nakamura J, et al. Spatiotemporal ATP Dynamics during AKI Predict Renal Prognosis. J Am Soc Nephrol 2020;31:2855-69.

16. Wei Q, Sun H, Song S, et al. MicroRNA-668 represses MTP18 to preserve mitochondrial dynamics in ischemic acute kidney injury. J Clin Invest 2018;128:5448-64.

17. Li HB, Zhang XZ, Sun Y, et al. HO-1/PINK1 Regulated Mitochondrial Fusion/Fission to Inhibit Pyroptosis and Attenuate Septic Acute Kidney Injury. Biomed Res Int 2020;2020:2148706.

18. Hall AM, Schuh CD. Mitochondria as therapeutic targets in acute kidney injury. Curr Opin Nephrol Hypertens 2016;25:355-62.

19. MacMillan-Crow LA, Mayeux PR. Female mice exhibit less renal mitochondrial injury but greater mortality using a comorbid model of experimental sepsis. Intern Med Rev (Wash D C) 2018. doi: 10.18103/imr.v4i10.768.

20. Liu JX, Yang C, Zhang WH, et al. Disturbance of mitochondrial dynamics and mitophagy in sepsis-induced acute kidney injury. Life Sci 2019;235:116828.

21. Zhou H, Toan S. Pathological Roles of Mitochondrial Oxidative Stress and Mitochondrial Dynamics in Cardiac Microvascular Ischemia/Reperfusion Injury. Biomolecules
2020;10:85.

22. Amin MM, Rafiei N, Poursafa P, et al. Association of benzene exposure with insulin resistance, SOD, and MDA as markers of oxidative stress in children and adolescents. Environ Sci Pollut Res Int 2018;25:34046-52.

23. Jomova K, Valko M. Advances in metal-induced oxidative stress and human disease. Toxicology 2011;283:65-87.

24. Tonnus W, Linkermann A. Gasdermin D and pyroptosis in acute kidney injury. Kidney Int 2019;96:1061-3.

25. Wang $\mathrm{W}, \mathrm{Zhu} M, \mathrm{Xu} Z$, et al. Ropivacaine promotes apoptosis of hepatocellular carcinoma cells through damaging mitochondria and activating caspase-3 activity. Biol Res 2019;52:36.

26. Zhang XJ, Mei WL, Tan GH, et al. Strophalloside induces apoptosis of SGC-7901 cells through the mitochondriondependent caspase-3 pathway. Molecules 2015;20:5714-28.

27. Sarathbabu S, Marimuthu SK, Ghatak S, et al. Induction of Apoptosis by Pierisin-6 in HPV Positive HeLa and HepG2 Cancer Cells is Mediated by the Caspase-3 Dependent Mitochondrial Pathway. Anticancer Agents Med Chem 2019;19:337-46.

28. Chen D, Zheng X, Kang D, et al. Apoptosis and expression of the Bcl-2 family of proteins and $\mathrm{P} 53$ in human pancreatic ductal adenocarcinoma. Med Princ Pract 2012;21:68-73.

29. Zhang $Y$, Yang $X$, Ge $X$, et al. Puerarin attenuates neurological deficits via $\mathrm{Bcl}-2 / \mathrm{Bax} /$ cleaved caspase-3 and Sirt3/SOD2 apoptotic pathways in subarachnoid hemorrhage mice. Biomed Pharmacother 2019;109:726-33.

30. Mahmoud AR, Ali FEM, Abd-Elhamid TH, et al. Coenzyme Q10 protects hepatocytes from ischemia reperfusion-induced apoptosis and oxidative stress via regulation of Bax/Bcl-2/PUMA and Nrf-2/FOXO-3/Sirt1 signaling pathways. Tissue Cell 2019;60:1-13.

(English Language Editor: J. Chapnick)
Cite this article as: Zhao L, Tian L, Wang S, Yang W, Lu X, Zhu C. Levosimendan in rats decreases acute kidney injury after cardiopulmonary resuscitation by improving mitochondrial dysfunction. Transl Androl Urol 2021;10(7):3010-3020. doi: $10.21037 /$ tau-21-443 